\title{
SYNTHETICALLY-FOCUSED SURFACE-PENETRATING RADAR FOR OPERATION FROM A MOVING VEHICLE
}

\author{
R. Benjamin, G. Hilton, R. Nilavalan, S. Litobarski, E. McCutcheon \\ University of Bristol, UK
}

Introduction. In the 1976 Edinburgh Conference, Benjamin [1] discussed near-field microwave focusing and $3 \mathrm{D}$ electronic scanning from a phased array, primarily for in-contact operation through an indexmatching soft pad, adjusting the operating frequency and impedance match to a given, fixed wavelength on the array - and thus in the medium. In [2] he then introduced the further novel concept of post-reception synthetic focusing, i.e. pulsing the array elements, one at a time, each time recording the signals received by all other relevant elements during the appropriate time window. A full 3D scan can then be synthesised retrospectively, from the total of all the data so recorded. These techniques were also covered by patents $[3,4]$.

This paper reports on a follow-on research programme, started recently under DERA sponsorship, on applying synthetic focusing to an oblong horizontal stand-off array, where all the voxels in a vertical slice underneath the centre-line of the array are synthesised electronically, with the third dimension provided by the search vehicle's forward movement. This should permit a very high search rate for buried mines, in a wide range of ground conditions.

\section{PERFORMANCE LIMITS}

Resolution. Any target $3 \mathrm{D}$ resolution cell on the surface will be in the field of view of all antenna elements within the beam-width of the (dipole) elements, and for a buried target the number of contributing antenna elements is further increased by the continued convergence of the cone of relevant paths, although the in-ground convergence angle $\theta$ (in the $\mathrm{X} / \mathrm{Z}$ and $\mathrm{Y} / \mathrm{Z}$ planes) is reduced by refraction, approximately as $1 / \sqrt{ } \varepsilon$, where $\varepsilon$ is the permittivity of the soil. See Fig. 1.

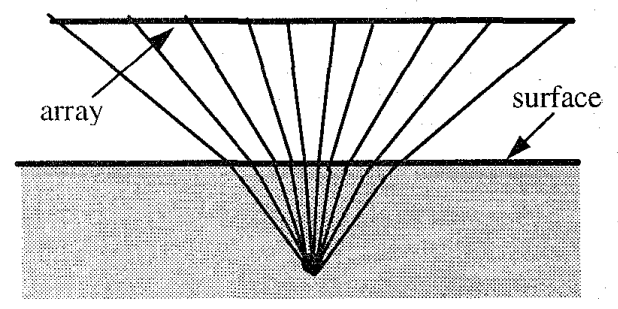

Fig 1 Refracted convergence

Since the wavelength in the ground is reduced by the same factor, the lateral resolution, $\lambda \theta, \sim 0.7 \lambda$ for an in- air convergence angle of $\pm 45^{\circ}$, is virtually independent of the refractive index. This has been confirmed by FDTD simulation [5]. However the depth of focus, $2 \lambda \theta^{2}$, increases as $\sqrt{ } \varepsilon$, i.e. the depth resolution is degraded, but only up to the point where it is limited by the pulselength in the ground, $n \lambda_{a} /(2 \sqrt{\varepsilon})$, where $n$ is the number of cycles in the pulse length and $\lambda_{\mathrm{a}}$ is the wavelength in air.

Thermal-noise limitations. At the same frequency and power as "conventional" ground-penetrating radar (GPR), the operation would be less thermal-noise limited, because:-

- The power per transmit element can be similar to that of a conventional GPR

- With synthetic focusing [2], the transmit power of all antenna elements "seeing" a given resolution cell contributes to the power received by all elements seeing that cell.

- We benefit from the coherent-processing gain due to all antenna elements pairs that have the given resolution cell within their field of view.

- The received signal and noise, from all distinct paths from any element via a given resolution cell to any other element, can be weighted selectively in accordance with their expected signal/noise and signal/clutter ratios[2].

At $1.4 \mathrm{GHz}$ (the only frequency at which suitable data were available), the estimated noise-limited maximum depth of burial, listed in table 1 , proved to be critically dependent on moisture, but almost independent of the soil type. (It is also independent of the stand-off distance of the antenna, since this does not alter the synthesised convergence angle.)

\begin{tabular}{|c|c|c|c|}
\hline moisture & $10 \%$ & $30 \%$ & $50 \%$ \\
\hline burial depth $(\mathrm{mm})$ & 600 & 330 & 275 \\
\hline
\end{tabular}

Table 1. Noise-limited burial depths

Clutter effects. Thus detectability will often be limited by back-scatter at discontinuities in refractive index at the surface, or from embedded pebbles etc. Clutter can only affect a given 3D resolution cell, C - see Fig. 2 - if it arrives at the receiver concurrently with the wanted signal. Hence it must arise within the common-pathlength locus: the ellipsoid of revolution - drastically flattened in the ground due to refraction - with the transmit and receive elements $\mathrm{A}$ and $\mathrm{B}$ as foci, which passes through point $C$ and has a thickness equivalent to \pm half a pulse length. $X$ represents a typical location of potential volume clutter within this locus shell. 


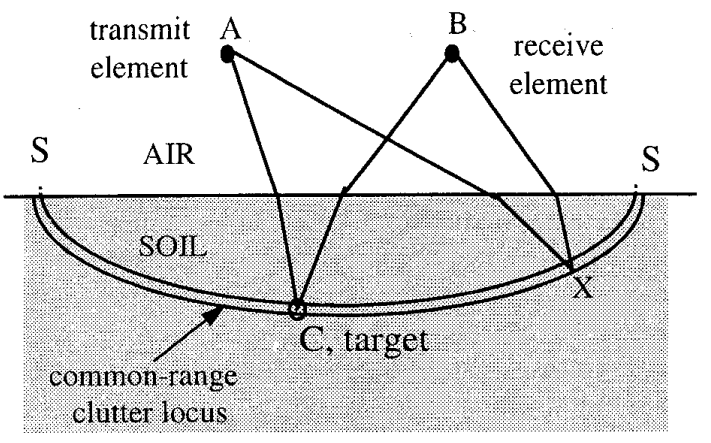

Fig. 2 Common-range clutter locus

Volume clutter. The volume clutter associated with a given path comes the reflections from the pebbles or other dielectric discontinuities from that part of the common-range "shell" of Fig. 2 which is within the field of view of both antenna elements. Thus the aggregate volume clutter arises from the non-coherent combination of these returns, from all the commonrange clutter shells relevant to the given focal cell, see Fig. 3.

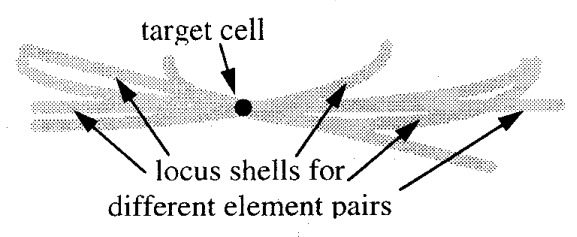

Fig. 3.

Coherent focusing and non-coherent clutter due to intersecting common-range loci.

Surface clutter For deeply buried objects, the volume clutter, like the wanted signal from the target cell $\mathrm{C}$ itself, is attenuated due to the penetration of lossy ground. Hence, the most troublesome effect is then likely to be that of surface clutter, from the elliptical foot-print where the above locus cuts the surface, corresponding to the two points $S$ in Fig. 2, but the width of this elliptical "race track" is determined by the pulse length in air. This surface clutter arises from any part of this "race-track" locus which is within the fields of view of both A and B, and where the back-scatter angular pattern of the ground would couple A to B.

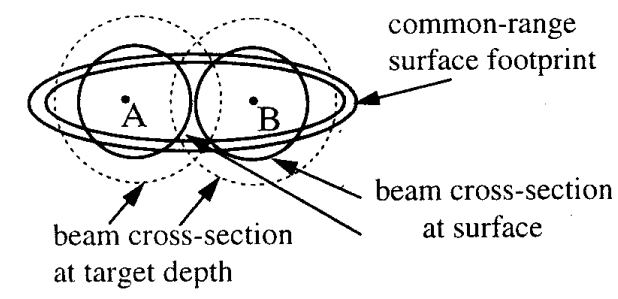

Fig. 4 Two Elements with no commonmain-beam surface clutter
The beam from an antenna element continues to diverge after ground penetration, albeit at a reduced rate, due to refraction. For a deeply buried object, the beam cross section at ground penetration is therefore substantially less than that at the depth of the object. Hence, as illustrated in Fig. 4 - and confirmed in computer simulations [5] - such overlap will normally only arise in a relatively small minority of element pairs (those situated close to each other). For optimum coherent summation, the signals from all distinct paths should be weighted by the estimated signal amplitude divided by the estimated clutter (or noise) power [6]. The relevant clutter power is the integral, over the "race-track", of the gains (and spreading losses) of the transmit and the receive antenna elements involved and of the appropriate directional back-scatter coefficient of the ground. With shallow depths of mine burial - or with soil of very high refractive index - there will be little widening of the beam cross section after ground penetration, and so a significant fraction of two-way paths may then include some surface clutter in the field of view of both antenna elements, and so the signals from these paths have to be scaled down, thus reducing their contribution to the processing gain.

Synthetic focusing is unique in its ability to weight the $\mathrm{N}(\mathrm{N}-1) / 2$ distinct 2-way paths, arising from $\mathrm{N}$ elements in view of an object, selectively, independently and optimally, rather than at best, merely weighting $\mathrm{N}$ transmit and $\mathrm{N}$ receive elements. This, together with the normal multi-element processing gain, should significantly widen the range of scenarios in which it can operate.

The effect of pebbles in the path. System performance is also affected by any pebbles etc. which are. in the path of the wanted signals. Pebbles small compared to the wavelength in the ground can probably be disregarded. However, the wavelength in the ground will be significantly smaller than that in air. Pebbles above this threshold can, for our purpose, be re presented by an absorbent disc, normal to the direction of radiation, of cross section equal to that of the pebble, see Fig. 5, where the black lumps represent pebbles. The resulting loss is roughly $\left(\mathrm{A}_{\mathrm{C}} / \mathrm{A}\right)^{2}$, where:

$A$ is the solid angle: focal-cell-to-active part of array, and $\mathrm{A}_{\mathrm{c}}$ ( $\mathrm{c}$ for clear) is the fraction of $\mathrm{A}$ that does not contain any pebble or pebbles.

Since the proportion of the previously usable beam cross section blocked, per unit depth, is constant, this results an appropriate increase in the attenuation coefficient, and so will reduce the maximum tolerable depth of burial. 


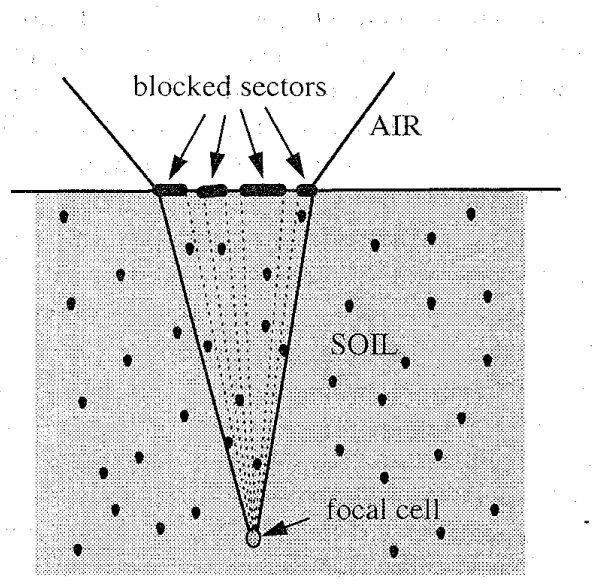

Fig. 5. Pebbles blocking part of coverage solid angle

The effects of surface features. The following mechanisms will introduce systematic errors in the estimated positions of the focal cells, but with little if any loss of gain or focal quality:-

- Inaccurate value of the refractive index (probably estimated from the ground reflections);

- Refractive index varying according to a smoothlytapered vertical profile;

- Stratified ground. In this case, however, the path loss - and the clutter - are increased by reflections at the inter-strata boundaries.

- An smooth air-ground boundary which is neither flat nor level, but which, within the surface patch penetrated by the relevant subset of $\mathrm{N}(\mathrm{N}-1) / 2$ paths, can be represented by the combination of a shallow (concave or convex) spherical curvature and modest $\mathrm{X}$ and $\mathrm{Y}$ gradients. This could however lead to gaps in the search coverage.

Any other macro-scale surface features could cause significant defocusing. Turning to the micro-scale, if the surface is rough, with a grain size which is not negligible compared to the wavelength, surface penetration will generate phase-noise which, in the limit, could prevent coherent processing.

Reverberation. If "multiple-bounce" echoes between the ground and the array should interfere with the detection of buried objects, it may be possible to measure the equivalent multiple-bounce echoes at neighbouring locations, and to cancel them coherently, provided the local air-ground geometry is sufficiently consistent. Alternatively we can insert an attenuating layer, of loss $\mathrm{L} \mathrm{dB}$, in front of the antenna. Thus the wanted signal and the related clutter are attenuated by $2 \mathrm{~L} \mathrm{~dB}$, but the double-bounce reverberation is attenuated by yet a further $2 \mathrm{~L} \mathrm{~dB}$ relative to this, and so should be rendered innocuous.
Non-coherent clutter cancellation. 'When surface roughness (or any other disturbance) prevents coherent focusing, there is still significant scope for non-coherent processing.

In Fig. 6, let $R_{i}$ be the resultant of the signal vector $S_{i}$ and the aggregate clutter vector $C_{i}$ of path $i$. Then $R_{i}>C_{i}$, or $R_{i}<C_{i}$, or $R_{i} \approx C_{i}$, depending on the phase relation between $S_{i}$ and $C_{i}$.

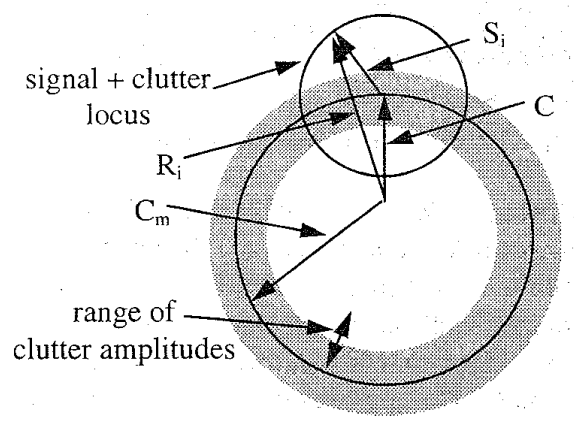

Fig. 6 Non-coherent clutter relationships

However, signals are only detectable when they are distinct from $\mathrm{C}_{\mathrm{m}}$, the mean value of the clutter (or noise) in the immediately surrounding resolution cells. Hence, for a detectable signal, $\left(\mathrm{C}_{\mathrm{i}}\right)_{\text {mean }} \approx \mathrm{C}_{\mathrm{m}}$.

Hence $\sum\left\{\left|R_{i}\right|-\left|C_{m}\right|\right\}$, over the $n(n-1) / 2$ contributing paths, should provide a non-coherent processing gain $\approx n / \sqrt{2}$.

Thus the spatial resolution is essentially preserved, and the $\mathrm{dB}$ processing gain against both thermal noise and clutter, albeit approximately halved, is still significant.

\section{IMPLEMENTATION CONSIDERATIONS}

Search rate. The propagation delays are so short - and signal-processing is so fast - that the search rate is limited only by the operation of the vehicle. When driving at 50 $\mathrm{km} / \mathrm{hr}$ along the edge of an already cleared area, with the array projecting laterally to scan a $2 \mathrm{~m}$ strip of the uncleared area, the search rate could thus be $10^{5} \mathrm{~m}^{2} / \mathrm{hr}$, reduced by any time taken to mark and/or clear real or suspected mines. When advancing into an uncleared area, the array would be mounted on a boom ahead of the vehicle. Once again a swathe $2 \mathrm{~m}$ wide would be cleared. The vehicle's speed would however be limited by the need to be able to stop before the vehicle is over the mine. Assuming instantaneous reaction to a suspect mine, a boom extension of $5 \mathrm{~m}$ and a braking rate of $0.25 \mathrm{G}$, would permit a speed of $18 \mathrm{~km} / \mathrm{hr}(5 \mathrm{~m} / \mathrm{s})$. With a tracked vehicle, a braking rate of; say, $1 \mathrm{~g}$ would be achievable, permitting a vehicle speed of $36 \mathrm{~km} / \mathrm{hr}-50 \mathrm{~km} / \mathrm{hr}$ for a $10 \mathrm{~m}$ boom. The shock of a sudden $1 \mathrm{~g}$ deceleration might however set off the mine! 
Time relationships. To generate independent verticalslice pictures at $10 \mathrm{~cm}$ spacing, for a vehicle speed of $50 \mathrm{~km} / \mathrm{hr}$, allows $7.2 \mathrm{~ms}$ to process the data from one set of transmissions by all elements. The time to collect the relevant information is however more limited:- To avoid the need for motion compensation, no two-way path delay must change by more than a small fraction of a cycle during the collection of the data on any resolution cell. This permits a vehicle advance of $0.1 \lambda$, giving a resolved component along the ray path of $a t$ worst $0.07 \lambda$. For $\lambda=25 \mathrm{~cm}$ and a vehicle speed of 50 $\mathrm{km} / \mathrm{hr}$, the coherence time is then $1.8 \mathrm{~ms}$. Working out detailed schedules shows that both times can readily accommodate all requisite operations. (However, the speed of A-to-D conversion required is close to the limit of the "state-of-the art".)

\section{CONCLUSIONS.}

1. Near-field spot-focusing from a non-contact phased array can give a high processing gain.

2. It also yields a high resolution, independent of soil characteristics.

3. Electronic scanning in a single vertical plane can be combined with mechanical scanning by the motion of the search vehicle.

4. Post-detection synthetic focusing permits a single pulse from each element to contribute to the observation of all resolvable $3 \mathrm{D}$ resolution cells.

5. Furthermore, it permits distinctive weighting for the paths for all distinct pairings of antenna-elements, according to their distinctive signal, noise and clutter characteristics.

6. Jointly, these features should allow the system operate in a wider range of scenarios than other radar-based scheme.

\section{ACKNOWLEDGEMENT}

This work has been carried out with the support of the Defence Evaluation and Research Agency.

(C) British Crown Copyright 1998.

\section{REFERENCES}

1. Benjamin, R. Near-field spot-focused microwave sensing for the detection of buried land-mines, IEE/EUREL Conference on the Detection of Buried Land-Mines, Edinburgh, October 1996.

2. Benjamin, R. Post-detection synthetic focusing in near-field radar, IEE/EUREL Conference on the Detection of Buried Land-Mines, Edinburgh, October 1996.

3. Benjamin, Near-Field Spot-Focused Microwaves, European Patent GB 9611801.3; 6/6/1996.

4. Benjamin, Synthetic Post-Reception Focusing, European Patent GB 9611800.5; 6/6/1996.

5. Microwave Detection of Sub-surface Objects, 2nd Progress report to DERA, Contract CB/LSC/20324/395, University of Bristol, May 1998.

6. Benjamin, Modulation, Resolution and Signal Processing in Radar and Sonar Pergamon, 1966. 